\title{
Relationship between Facet Joint Asymmetry and Adjacent Intervertebral Disc Degeneration in Lumbar Disc Herniation
}

\author{
Shenghui Huang \\ Department of Orthopedics, The Seventh Affiliated Hospital of Sun Yat-sen University, Shenzhen, China \\ Email: shenghuihuang@126.com
}

How to cite this paper: Huang, S.H. (2020) Relationship between Facet Joint Asymmetry and Adjacent Intervertebral Disc Degeneration in Lumbar Disc Herniation. Journal of Biosciences and Medicines, 8, 117123.

https://doi.org/10.4236/jbm.2020.811011

Received: October 12, 2020

Accepted: November 14, 2020

Published: November 17, 2020

Copyright $\odot 2020$ by author(s) and Scientific Research Publishing Inc. This work is licensed under the Creative Commons Attribution International License (CC BY 4.0).

http://creativecommons.org/licenses/by/4.0/

\begin{abstract}
Objective: To analyze the relationship between facet joint asymmetry and adjacent intervertebral disc degeneration in lumbar disc herniation. Methods: Fifty patients with L4/5 lumbar disc herniation were enrolled in the study. All patients underwent lumbar MRI examination, to compare the facet asymmetry of lumbar disc herniation and the degeneration of cartilage endplate and intervertebral disc of adjacent segments, and analyze the relationship between them. Results: There was no significant correlation between L4/5 facet asymmetry and L5/S1 intervertebral disc degeneration score and cartilage endplate degeneration score $(P>0.05)$. L4/5 facet asymmetry was significantly correlated with $\mathrm{L} 3 / 4$ disc degeneration score $(P<0.01)$, but not with cartilage endplate degeneration score $(P>0.05)$. Conclusion: There was a positive correlation between the facet joint angle of lumbar disc herniation and the degeneration of upper proximal segment intervertebral disc. Early correction of lumbar facet angle asymmetry can provide beneficial guidance for further prevention and treatment.
\end{abstract}

\section{Keywords}

Lumbar Disc Herniation, Facet Asymmetry, Adjacent Segment Degeneration

\section{Introduction}

Lumbar disc herniation is a common clinical disease with intervertebral disc degeneration as the main pathological change. Some patients with lumbar disc herniation may have degenerative changes in adjacent segments. L4/5 and L5/S1 are the segments that maintain spinal stability and activity, and bear about $1 / 5$ of the body weight. L4/5 segment is the most common site of lumbar disc hernia- 
tion. Facet joint asymmetry and adjacent disc degeneration play an important role in the pathophysiology of intervertebral disc herniation. Lumbar facet joint asymmetry has been the research focus in spinal degenerative diseases [1] [2] [3], L4 and L5 lumbar facet joint asymmetry is the most common [4], while there is few study concerning about the relationship between facet joint asymmetry and adjacent intervertebral disc degeneration in lumbar disc herniation. In this study, MRI was used to observe the imaging features of L4/5 lumbar disc herniation and the adjacent intervertebral discs of L3/4 and L5/S1.

\section{Data and Methods}

\subsection{General Information}

50 patients with L4/5 disc herniation in the seventh Affiliated Hospital of Sun Yat sen University were selected from May 2018 to September 2020, including 33 males and 17 females. The inclusion criteria were "guidelines for diagnosis and treatment of lumbar disc herniation" formulated by the spine surgery group of orthopedics, Chinese Medical Association in 2020, excluding patients with lower extremity arthritis and lumbar tumor, as well as lumbar scoliosis, lumbar spondylolisthesis, lumbar compression fractures and other spinal deformities.

\subsection{Method}

All patients underwent lumbar MRI examination before operation, which were operated and read by orthopedic doctors with rich clinical experience.

\subsection{Observation Index}

The cartilage endplate and intervertebral disc degeneration of L3/4 and L5/ S1 discs were compared. Cartilage endplate and intervertebral disc were evaluated as follows. 1) Pappou grade [5] was used as the evaluation standard of cartilage endplate degeneration. According to the T1 image of MRI, the shape of endplate can be divided into flat type, concave type and irregular type, that is to draw a straight line at the anterior and posterior edge of the vertebral body along the median sagittal position of the endplate. Concave type refer to the intervertebral disc tissue between the edge of the endplate and the straight line, flat type refer to the edge of the endplate coincides with the straight line, and irregular type refer to the vegetation outside the line. Irregular type is grade III, with 3 points; flat type is grade II, accounting for 2 points; concave type is grade I, accounting for 1 point. The higher the score, the more serious the degeneration. 2) Pfirmann grading [6] was used as the standard of intervertebral disc degeneration, which was divided into A-E grades. Grade A:the structure of intervertebral disc showed uniform white high signal, and the height of intervertebral disc was normal; Grade B: intervertebral disc showed uneven white high signal, the difference between annulus fibrosus and nucleus pulposus was obvious, with or without horizontal gray band; Grade C: intervertebral disc structure was uneven, with gray signal intensity in the middle, the difference between annulus fibrosus 
and nucleus pulposus was not obvious, and the height of intervertebral disc was normal or slight; Grade D: intervertebral disc structure was uneven and showed black low signal, the difference between nucleus pulposus and annulus fibrosus disappeared, and the height of intervertebral disc was normal or moderately decreased; Grade E: intervertebral disc structure was uneven, showing black low signal; the difference between nucleus pulposus and annulus fibrosus was lost, and there was intervertebral space collapse, 1 - 5 points for A-E grade. The higher the score, the more serious the degeneration. 3) The horizontal cross-sectional images of L4/5 segment upper endplate were obtained, and the front and rear ends of facet joint surface were connected. The angle between the line and the central sagittal line of the vertebral body was the lumbar facet angle. The angle value of the left joint $(\alpha)$ and the right facet joint angle value $(\beta)$ were recorded (as shown in Figure 1). The angle difference $|\alpha-\beta|$ was used as the quantitative index of facet asymmetry.

\subsection{Data Analysis}

Statistical software SPSS 23.0 was used for analysis and processing. The measurement data were expressed as $(\bar{X} \pm S)$. Paired t-test was used to compare the unilateral lumbar facet joint angle of two segments; Spearman grade correlation analysis was used to analyze the cartilage endplate and intervertebral disc degeneration score between herniated and adjacent segments, with test level $\alpha=0.05$.

\section{Results}

1) Comparison of the difference of bilateral facet joint angle of L4/5 lumbar spine $(\bar{X} \pm S, \mathrm{n}=50$ ), there was significant difference between left and right lumbar facet angle in two segments $(P<0.05)$, As shown in Table 1.

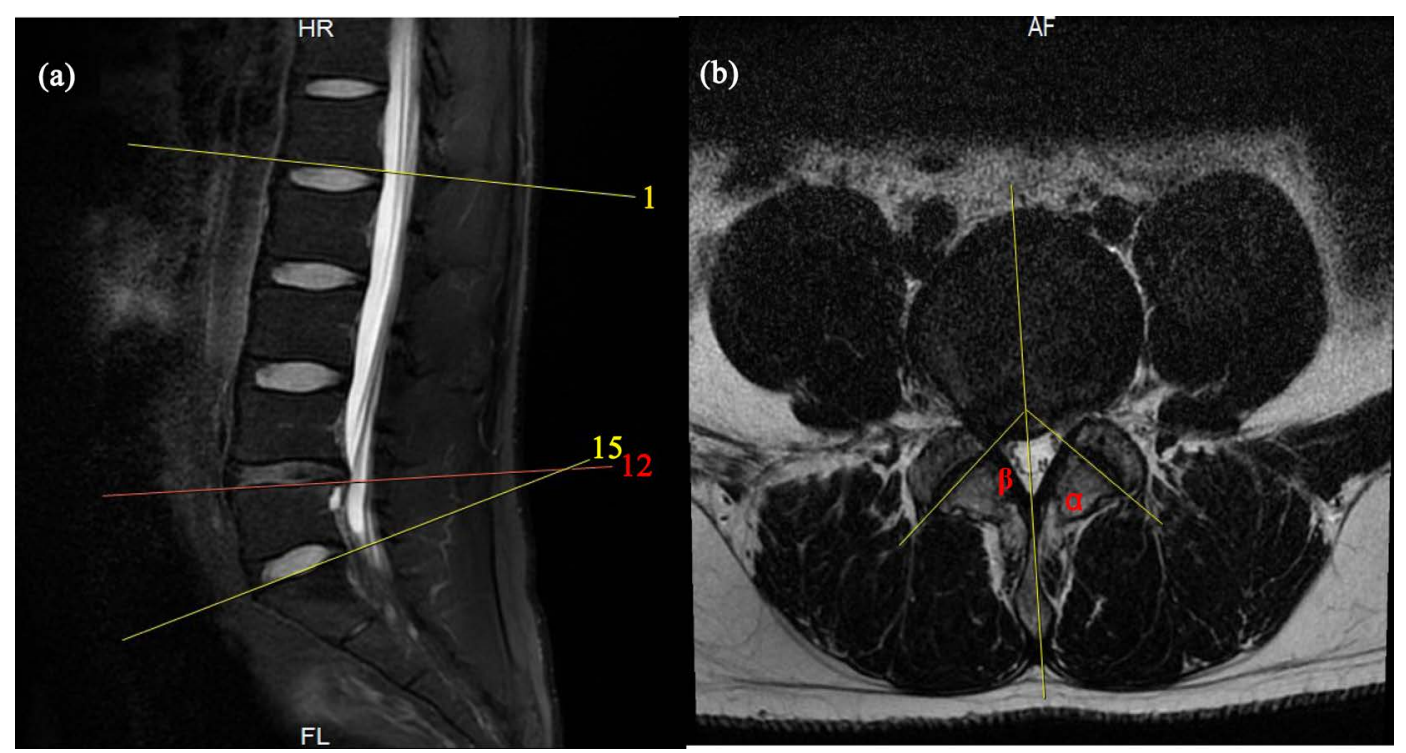

Figure 1. (a) shows the upper endplate plane of L5 vertebral body in the median sagittal position; (b) shows the right and left lumbar facet joint angles $(\angle \beta, \angle \alpha)$ in $L 4 / 5$ cross section. 
2) There was no correlation between L3/4, L5/S1 cartilage degeneration and L4/5 lumbar facet angle difference ( $P>0.05)$, as shown in Table 2.

3) There was a positive correlation between L3/4 disc degeneration and L4/5 lumbar facet angle asymmetry $(P<0.01)$, there was no correlation between $\mathrm{L} 4 / 5$ lumbar facet angle asymmetry $(P>0.05)$ (Table 3$)$.

\section{Discussion}

MRI is a kind of tomographic imaging technology. Due to the different composition of intervertebral disc, the signal display of cartilage endplate, nucleus pulposus and annulus fibrosus on MRI are also significantly different. MRI has the same three-dimensional scanning function as $\mathrm{CT}$, and has obvious advantages in the identification of soft tissue, especially in the early stage of inflammation. Therefore, MRI has become the first choice for the diagnosis of degenerative changes of intervertebral disc [7]. Intervertebral disc degeneration is a kind of degenerative disease with biomechanical and morphological changes caused by degradation of extracellular matrix and cytoplasmic loss. The occurrence is

Table 1. Comparison of the difference of bilateral facet joint angle of L4/5 lumbar spine.

\begin{tabular}{cc}
\hline & $\mathrm{L} 4 / 5(\mathrm{n}=52)$ \\
\hline$\alpha\left(^{\circ}\right)$ & $44.25 \pm 11.06$ \\
$\beta\left(^{\circ}\right)$ & $44.32 \pm 10.78$ \\
& $\mathrm{t}=9.823, P<0.05$ \\
\hline
\end{tabular}

Table 2. The relationship between facet joint asymmetry and adjacent cartilage degeneration.

\begin{tabular}{ccc}
\hline & $\mathrm{L} 3 / \mathbf{4}(\mathrm{n}=\mathbf{5 0})$ & $\mathrm{L} 5 / \mathrm{S} 1(\mathrm{n}=\mathbf{5 0})$ \\
I & 28 & 21 \\
II & 19 & 20 \\
III & 3 & 9 \\
& $\mathrm{r}=0.231, P>0.05$ & $\mathrm{r}=0.019, P>0.05$ \\
\hline
\end{tabular}

$r$ is the correlation coefficient of Spearman analysis.

Table 3. The relationship between facet joint asymmetry and adjacent intervertebral disc degeneration.

\begin{tabular}{ccc}
\hline & L3/4 ( $=\mathbf{5 0})$ & L5/S1 ( $=\mathbf{5 0})$ \\
\hline A & 14 & 11 \\
B & 12 & 11 \\
C & 13 & 16 \\
D & 11 & 9 \\
E & 0 & 3 \\
& $\mathrm{r}=0.444, P<0.01$ & $\mathrm{r}=0.090, P>0.05$ \\
\hline
\end{tabular}

$r$ is the correlation coefficient of Spearman analysis. 
mainly related to nutritional factors, mechanical factors and apoptosis factors of intervertebral disc [8]. It was found that there were different degrees of degenerative changes in the MRI images of patients with lumbar disc herniation. The main manifestations were low signal of intervertebral disc in T2 weighted images, mild disc bulging, low intervertebral disc height and cartilage endplate sclerosis [9]. The degeneration of adjacent intervertebral disc is related to the change of lumbar stress distribution and exercise load. The degeneration of herniated intervertebral disc significantly increases the stress and internal pressure load of adjacent intervertebral disc, especially the adjacent segment with degeneration, which can further accelerate the process of degeneration; when the pressure in adjacent intervertebral disc increases and the stress of annulus fibrosus and lamina increases, the deformation of responsible segment will act on the adjacent segment and cause degeneration.

Lumbar facet joint asymmetry is a hot research topic in recent years. When lumbar facet joint asymmetry occurs, it will lead to abnormal load mode, which increases the risk of spinal rotation instability and intervertebral disc degeneration [10] [11]. Do DH et al. [12] confirmed that facet joint asymmetry leads to increased biomechanical load of patients, resulting in increased disc herniation. However, the relationship between lumbar asymmetry and adjacent lumbar disc degeneration in patients with lumbar disc herniation has not been reported.

The results showed that the facet angle asymmetry of L4/5 lumbar disc herniation was positively correlated with the degeneration of upper proximal segment, but not with L5/S1 disc degeneration. There was no relationship between facet angle asymmetry of L4/5 lumbar disc herniation and degeneration of upper and lower cartilage endplates.

The possible reasons are as follows: 1) the asymmetry of lumbar facet joints causes the inflammation of facet joints. Due to the mechanical rotation and other forces, the metabolic products and nutrient supply of intervertebral disc are out of balance, resulting in the dysfunction of intervertebral disc cells, and then leading to degenerative changes of lumbar intervertebral disc. 2) Previous studies have reported that L5/S1 intervertebral disc degeneration is more prone to degeneration than L3/4 segment. This study found that intervertebral disc degeneration of upper segment has stronger correlation with lumbar facet joint asymmetry, which may be related to the biomechanical characteristics of different segments. The pressure in the upper intervertebral disc is higher than that in the lower intervertebral disc, combined with the influence of body mass, age and other factors, the degree of degeneration of the upper intervertebral disc is more serious than that of the lower segment. Studies have shown that upper intervertebral disc degeneration is more likely to occur after lumbar internal fixation [13] [14]. 3) Cartilage endplate degeneration is caused by many factors, and the abnormal stress caused by articular process joint asymmetry is not enough to cause significant cartilage endplate degeneration. In addition, the number of cases in this study is limited, and the influence of age, weight, gender and other 
factors on the results cannot be excluded.

This study is a retrospective study, and the clinical sample size is too small, which has certain limitations, and needs to be further studied.

\section{Conflicts of Interest}

The author declares no conflicts of interest regarding the publication of this paper.

\section{References}

[1] Xu, W.B., et al. (2016) Facet Orientation and Tropism: Associations with Asymmetric Lumbar Paraspinal and Psoas Muscle Parameters in Patients with Chronic Low Back Pain. Journal of Back and Musculoskeletal Rehabilitation, 29, 581-586. https://doi.org/10.3233/BMR-160661

[2] Jelec, V., et al. (2016) Facet Orientation and Tropism: Association with Accelerated Degeneration of Stabilizing Structures in Lower Lumbar Spine. Acta Clinica Croatica, 55, 117-124. https://doi.org/10.20471/acc.2016.55.01.17

[3] Wang, H. and Zhou, Y. (2016) Facet Tropism: Possible Role in the Pathology of Lumbar Disc Herniation in Adolescents. Journal of Neurosurgery Pediatrics, 18, 111-115. https://doi.org/10.3171/2015.7.PEDS15175

[4] Binder, D.S. and Nampiaparampil, D.E. (2009) The Provocative Lumbar Facet Joint. Current Reviews in Musculoskeletal Medicine, 2, 15-24. https://doi.org/10.1007/s12178-008-9039-y

[5] Pappou, I.P., Cammisa, F.J. and Girardi, F.P. (2007) Correlation of End Plate Shape on MRI and Disc Degeneration in Surgically Treated Patients with Degenerative Disc Disease and Herniated Nucleus Pulposus. The Spine Journal, 7, 32-38. https://doi.org/10.1016/j.spinee.2006.02.029

[6] Pfirrmann, C.W., Metzdorf, A., Zanetti, M., et al. (2001) Magnetic Resonance Classification of Lumbar Intervertebral Disc Degeneration. Spine (Phila Pa 1976), 26, 1873-1878. https://doi.org/10.1097/00007632-200109010-00011

[7] Pathria, M., Sartoris, D.J. and Resnick, D. (1987) Osteoarthritis of the Facet Joints: Accuracy of Oblique Radiographic Assessment. Radiology, 164, 227-230.

https://doi.org/10.1148/radiology.164.1.3588910

[8] Landi, A., Palmarini, V., D’Elia, A., et al. (2016) Magnetic Resonance Diffusion Tensor Imaging and Fiber-Tracking Diffusion Tensor Tractography in the Management of Spinal Astrocytomas. World Journal of Clinical Cases, 4, 1-4. https://doi.org/10.12998/wjcc.v4.i1.1

[9] Zhong, K.H., Xu, H.B. and Bai, S. (2015) Comparative Study on MRI and Pathological Changes of Lumbar Intervertebral Disc Degeneration. Journal of Xinxiang Medical College, 32, 314-317.

[10] Mahato, N.K. (2011) Facet Dimensions, Orientation, and Symmetry at L5-S1 Junction in Lumbosacral Transitional States. Spine (Phila Pa 1976), 36, E569-E573. https://doi.org/10.1097/BRS.0b013e3181f6ecb2

[11] Kim, H.J., Chun, H.J., Lee, H.M., et al. (2013) The Biomechanical Influence of the Facet Joint Orientation and the Facet Tropism in the Lumbar Spine. The Spine Journal, 13, 1301-1308. https://doi.org/10.1016/j.spinee.2013.06.025

[12] Do, D.H., Taghavi, C.E., Fong, W., et al. (2011) The Relationship between Degree of Facet Tropism and Amount of Dynamic Disc Bulge in Lumbar Spine of Patients Symptomatic for Low Back Pain. European Spine Journal, 20, 71-78. 
https://doi.org/10.1007/s00586-010-1558-8

[13] Gao, J. and Zhang, X. (2017) MRI Analysis of Intervertebral Disc Degeneration Adjacent to Fusion Segment after Lumbar Fusion. Chinese Journal of Bone and Joint Injury, 32, 68-69.

[14] Zhang, X.G., Zhang, J., Zhou, T., et al. (2018) MRI Features of Herniated Intervertebral Disc and Adjacent Intervertebral Disc Degeneration in Patients with Lumbar Disc Herniation. Journal of Practical Clinical Medicine, 22, 146-148. 